\title{
Lip lupus erythematosus
}

\author{
Johanna M Warren \\ Medical Student ${ }^{\top}$ \\ Peter F Bourke \\ PhD, FRACP, \\ Clinical Immunologist and \\ Allergist \\ Lachlan J Warren \\ MB BS, FRACGP, FACD \\ Visiting Dermatologist ${ }^{2}$ \\ IUniversity of Adelaide \\ Adelaide, SA. \\ 2 Royal Darwin Hospital \\ Darwin, NT. \\ johanna.warren@ \\ student.adelaide.edu.au
}

doi: 10.5694/mjal2.11518

\section{Clinical record}

A 40-year-old Indigenous Australian woman was referred to an outreach dermatology clinic in the Top End of the Northern Territory for assessment of painful lip ulceration of 2 years' duration. She had been reviewed 16 months earlier in a distant regional centre, and a diagnosis of squamous cell carcinoma of the lip had been proposed. Skin biopsy had not been performed and the patient had refused a planned extensive surgical excision of the lip lesion at that assessment. Her general health was good.

Examination at the outreach clinic showed extensive and bilateral lower lip exophytic ulceration and crusting (Box 1). The upper lip and other cutaneous and oral surfaces were normal.

Lower lip skin biopsy samples were taken from the ulcer and adjacent lower lip mucosa to confirm a clinical diagnosis of lupus erythematosus and to exclude malignancy (Box 2). The hyperkeratotic squamous epithelium ranged from atrophic to acanthotic, with foci of lichenoid basal vacuolar damage resulting in squamous cell apoptosis and colloid bodies.
The submucosa contained a dense mixed inflammatory cell infiltrate of lymphocytes, histiocytes, plasma cells and melanophages. Direct immunofluorescence investigation showed weak (1+) granular IgM and complement component 3 positivity along the junctional region. No squamous dysplasia or malignancy was identified.

The results of blood testing, including antinuclear antibody testing, extractable nuclear antigen and DNA-antibody studies, were negative. Serum complement levels were normal. The patient was instructed to reduce lip sun exposure, apply broadspectrum sunscreen twice daily and apply betamethasone dipropionate cream $(0.5 \mathrm{mg} / \mathrm{g})$ daily. On review 3 months later, there was a dramatic resolution of pain and ulceration. In the absence of any clinical or histological suggestion of malignancy, surgical treatment was not indicated or anticipated. • ip and oral ulceration may be a feature of systemic lupus erythematosus (SLE) or a manifestation of cutaneous discoid lupus erythematosus (DLE). ${ }^{1}$ Various studies have suggested a high incidence and prevalence of both SLE and DLE in Indigenous Australian populations, with both forms mostly affecting women. ${ }^{2-6}$ We have treated many patients with DLE on the lower lip, both as a solitary disease manifestation and as part of more disseminated cutaneous disease. In most cases the diagnosis had not been suspected by treating health practitioners. DLE needs to be considered as a potential cause for lip symptoms and ulceration in this situation, with differential diagnoses including infections (eg, candidiasis, syphilis and streptococcal infection), lichen planus, fixed drug reactions and actinic malignancies.

Clinical features that may prompt suspicion of lip DLE include red, friable and delicate skin in early phases, with progression to erosions, ulceration, crusting and pigment loss with time and chronicity. ${ }^{6}$ The reason for a seemingly higher rate of SLE and DLE in Indigenous Australians compared with non-Indigenous Australians is unclear. However, genetic predisposition (human leukocyte antigen and complement pathway associations) and prior infective and environmental (sunlight) triggers have been hypothesised. $^{2-4}$

DLE has characteristic histological features on skin biopsy, ${ }^{1}$ but selection of sites for biopsy is critical as epithelial destruction in areas of ulceration may result in loss of diagnostic findings. ${ }^{7}$ Clear communication to the pathologist about the purpose of the biopsy is essential, specifically whether exclusion of malignancy or confirmation of DLE is desired. For confirmation of suspected DLE, biopsy of clinically abnormal skin adjacent to but not involving areas of ulceration is recommended. If malignancy is suspected, taking multiple biopsies of the most indurated lesional skin is appropriate. Testing by direct immunofluorescence may be helpful but is less reliable than histopathological assessment in the diagnosis of
DLE, with low sensitivity. ${ }^{7,8}$ Additionally, sun-exposed skin from healthy patients often shows false positive results of immunofluorescence testing. ${ }^{9}$ There is an increased rate of squamous cell carcinoma in lesions of DLE, ${ }^{7}$ with epithelial dysplasia on biopsy reported as the prime indicator of risk of malignant transformation. ${ }^{10}$ An awareness of this association should prompt detailed searching and reporting of cytological atypia during histopathological assessment.

Management priorities for lip DLE include precise diagnosis, exclusion of systemic involvement and identification of triggers (including medications). In most patients with DLE the prognosis is good, with progression to SLE being uncommon. ${ }^{1}$ Methods to reduce ultraviolet exposure on affected areas, including seeking shade, direct sun avoidance and regular sunscreen use, are essential. Potent topical corticosteroid application will often bring about rapid improvement. If general and topical therapies fail, the patient's adherence should be reviewed. If systemic treatment is required, oral hydroxychloroquine is initially recommended. ${ }^{1}$

1 Lupus erythematosus of the lower lip, with ulceration and crusting of 2 years' duration, in a 40-year-old Indigenous woman

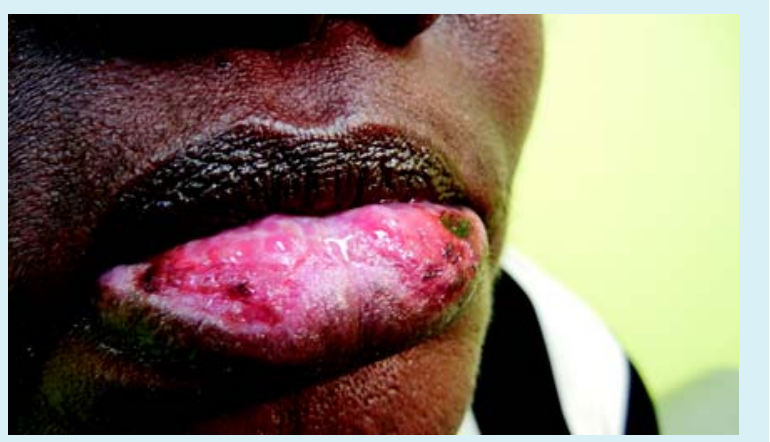




\section{Case reports}

2 Lower lip skin biopsy image* showing lichenoid cheilitis with vacuolar basal epithelial damage, lymphocyte exocytosis, epithelial atrophy and an intense inflammatory infiltrate ${ }^{\dagger}$

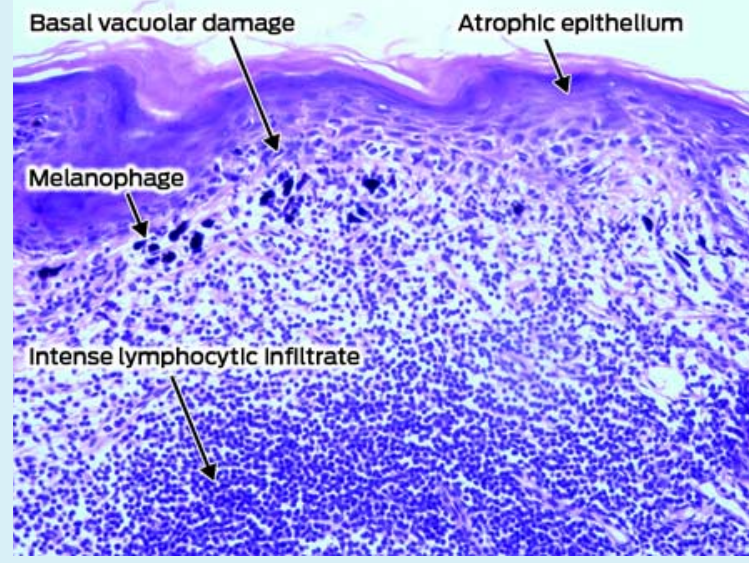

* Magnification × 100; sample stained with haematoxylin and eosin. $†$ No squamous dysplasia is present.

An awareness that the lower lip is a common site for lupus erythematosus, particularly in Indigenous Australian patients, will facilitate early diagnosis and appropriate management. A comprehensive lupus erythematosus database, including epidemiological, clinical, serological and outcome measures, is being established at Royal Darwin Hospital to provide further insights and enable statistical analysis.

\section{Lessons from practice}

- Lower lip ulceration and thickening is commonly caused by lupus erythematosus in northern Australia

- There is a high incidence of both systemic and cutaneous lupus erythematosus in Indigenous Australians

- Careful and informed skin biopsy technique and interpretation of histopathological findings are critical in differentiating causes of lower lip symptoms

Acknowledgements: We thank Craig James for his interpretation of pathology results and valued comments on this article.

Competing interests: No relevant disclosures.

1 Callen JP. Cutaneous lupus erythematosus: a personal approach to management. Australas J Dermatol 2006; 47: 13-27.

2 Anstey NM, Bastian I, Dunckley H, Currie BJ. Systemic lupus erythematosus in Australian Aborigines: high prevalence, morbidity and mortality. Aust NZ J Med 1993; 23: 646-651.

3 Bossingham D. Systemic lupus erythematosus in the far north of Queensland. Lupus 2003; 12: 327-331.

4 Grennan DM, Bossingham D. Systemic lupus erythematosus (SLE): different prevalences in different populations of Australian aboriginals. Aust NZ J Med 1995; 25: 182-183.

5 Segasothy M, Phillips PA. Systemic lupus erythematosus in Aborigines and Caucasians in central Australia: a comparative study. Lupus 2001; 10: 439-444.

6 Green A. Discoid erythematosus in Australian Aborigines. Australas J Dermatol 1995; 36: 175-177.

7 Weedon D. Weedon's skin pathology. 3rd ed. London: Churchill Livingstone Elsevier, 2010.

8 Williams RE, Mackie RM, O'Keefe R, Thomson W. The contribution of direct immunofluorescence to the diagnosis of lupus erythematosus. J Cutan Pathol 1989; 16: 122-125.

9 Fabré VC, Lear S, Reichlin M, et al. Twenty percent of biopsy specimens from sun-exposed skin of normal young adults demonstrate positive immunofluorescence. Arch Dermatol 1991; 127: 1006-1011.

10 Liu W, Shen ZY, Wang LJ, et al. Malignant potential of oral and labial chronic discoid lupus erythematosus: a clinicopathological study of 87 cases. Histopathology 2011; 59: 292-298. 